\title{
ASSOCIATED RISK FACTORS OF ACUTE MYOCARDIAL INFARCTION AMONG HUNAN POPULATION IN CHINA
}

\author{
Md Sayed Ali Sheikh \\ Department of Internal Medicine, Cardiology, College of Medicine, Jouf University, Sakaka, Aljouf, Kingdom of Saudi \\ Arabia.
}

Corresponding author: Md Sayed Ali Sheikh, E-mail: drsheikh07@hotmail.com

\begin{abstract}
The purpose of this study to determine the clinical characteristic of the associated risk factors of acute myocardial infarction patients (AMI) among the Hunan Han population in China. The retrospectively collected the records data of 595 both STEMI and NSTEMI patients from the first Xiangya hospital, Hunan, China over a period of January 2018 and December 2018. These studies revealed clinical characteristics with associated risk factors among acute myocardial infarction patients. A total of 595 diagnosed acute myocardial infarction patients participated in this study among males $70.9 \%$ and females $29 \%$ with mean age e $52.9+11.3$ years. While $90 \%$ had STEMI and $9.9 \%$ had NSTEMI. The chest pain $94.4 \%$, $86 \%$ and shortness of breath 55\%, 100\% presented with STEMI and NSTEMI groups respectively. Smoking incidence in male subject 70.3\% had higher than in female subject $29.1 \%(P<0.05)$. Hypertension and diabetes mellitus found $59 \%$, 69.5\% in male participants as compared to $40.6 \%, 30.4 \%$ in female participants respectively $(P<0.05)$. However, no statistical difference was found among dyslipidemia males $48.6 \%$ and females $47.4 \%$. The most common type of AMI was STEMI and usually presented with chest pain and shortness of breath. The AMI patients were more found in male and common associated risk factors were smoking and hypertension followed by diabetes mellitus and dyslipidemia.
\end{abstract}

Keywords: Acute myocardial infarction, ST elevation myocardial infarction, Chest pain, Smoking, Hypertension.

\section{INTRODUCTION}

In recent years cardiovascular disease dramatically increasing in developing countries. ${ }^{1}$ According to data from 2010 in china, approximately 8 million of the Chinese population who had been suffering a myocardial infarction (MI) over the age of 40 years and more than 1 million deaths occur per year. ${ }^{2,3}$ Therefore, the World Health Organization declares that the number of $\mathrm{Ml}$ will be increased by roughly 23 million by the 2030 years. Although another study reported in china around 290 million had CAD along with 11 million ischemic heart diseases in the year of 2015 (IHD). ${ }^{4}$

Many study review in the developed countries such as Canada, Europe, and the USA that myocardial infarction may depend on gender and age variation which was comparatively increased the female mortality rate than males. 5,6 However, published information from China Patient-centered Evaluative Assessment of Cardiac Events (China PEACE)- this Retrospective focus on acute myocardial infarction patients and as well as ST-Segment Elevation Myocardial Infarction (STEMI), the result shown gradually increasing both genders of STEMI patients admissions in hospital since the last decade. While females $30 \%$ was affected among total admission patients. ${ }^{7}$ Thus, it has been clearly documented that AMI may exist gender dependable and need proper arrangement which supports to improve the care of AMI patients. Conversely, since the last few decades, myocardial infarction plays a leading cause of the high mortality rate in china, which was considered a burden for the Chinese population. ${ }^{7}$

The myocardial infarction (MI) patients frequently complain about chest pain even in resting position. The electrocardiographic (ECG) changes are important to know the status of AMI that will help for proper management. However, the major risk factor for $\mathrm{AMI}$ as hypertension, diabetes mellitus, dyslipidemia, and as well as smoking. Previously reported that hypertension and diabetes have acted as a risk for developing myocardial infarction. ${ }^{8}$ Besides, another study recognized that diet, smoking, obesity behind the responsible for AMI. ${ }^{9}$ While in India the cigarette smoking is the highest causes of MI. ${ }^{10}$ Several study evidence of review of $2171 \mathrm{MI}$ patients and discovered the individual smoking has capacity to produce $\mathrm{Ml}$ and it was affected $37 \%$ among the total of the population in South Asia. ${ }^{11}$ On the other hand, some study focuses on common risk factors such as diabetes and hypertension, which are responsible for increasing the risk of Ml. Therefore present study aimed to evaluate the commonly associated risk factor among Ml in Chinese patients in Hunan China, which will help to prevent and proper management of MI, 
and may reduce the mortality and morbidity rate in China.

\section{METHODS}

This retrospective study was designed with a diagnosed of acute myocardial infarction subject who was conducted at the cardiac department of the First Xiangya Hospital, Central South University, Hunan, China, from January 2018 and December 2018. A total of five hundred ninety-five myocardial infarction patients participated in this study. The Hospital recorded data such as age, gender, smoking, hypertension, diabetes mellitus, dyslipidemia, and clinical patterns of STEMI and NSTEMI were collected from all the study subjects. The review of laboratory data including high-density lipoprotein (HDL), low-density lipoprotein (LDL), total cholesterol (TC), blood sugar level along with echocardiography (ECG) reports were also included. The ethical clearance approved by the Ethics Committee of Central South University.

\section{Statistical analysis}

The chi-square test was used for differences in risk factors of both males and females for categorical variables. All available data were analyzed by using SPSS 20.00 software with considering at significant at ( $p$-value of $<0.05)$.

\section{RESULTS}

A total number of 595 diagnosed $\mathrm{AMI}$ patients among 422 (70.9\%) males and 173 (29) \%) females have participated in this study. The mean age of all patients was $52.9+11.3$ years. The demographic characteristic of patients was represented in table1 .

Table 1. Demographic and clinical characteristics

\begin{tabular}{ll}
\hline Factors & $\begin{array}{l}\text { Total } \quad \text { number } \\
\text { patients }(\mathrm{n}=595) \%\end{array}$ \\
\hline Mean age & $52.9+11.3$ years \\
Male & $422(70.9)$ \\
Female & $173(29)$ \\
Total cholesterol & $4.2 \pm 0.9 \mathrm{mmol} / \mathrm{L}$ \\
LDL & $2.2 \pm 0.8 \mathrm{mmol} / \mathrm{L}$ \\
HDL & $1.3 \pm 0.3 \mathrm{mmol} / \mathrm{L}$ \\
Fasting Blood Sugar & $6.9 \pm 3.7 \mathrm{mmol} / \mathrm{L}$ \\
\hline
\end{tabular}

The greater part of AMI patients (90\%) shown elevation of ST-segment (STEMI), while only (9.9) had non-ST elevation MI (NSTEMI). The acute myocardial infarction more presents $51.3 \%$ in the anterior wall and $41.9 \%$ in the inferior wall. While
$1.6 \%, 2.7 \%$, and $2.23 \%$ occur in anterior inferior, anterior lateral and inferior lateral wall respectively were shown in Table-2.

Table 2. ECG findings among all participants in this study.

\begin{tabular}{ll}
\hline ECG findings & Number \% \\
\hline ST-segment elevation MI & $536(90)$ \\
Non- ST segment elevation MI & $59(9.9)$ \\
Pathological Q wave & $35(6.5)$ \\
Site of infraction (N=536) & \\
Anterior wall & $275(51.3)$ \\
Inferior wall & $225(41.9)$ \\
Anterior + inferior wall & $9(1.6)$ \\
Anterior + lateral wall & $15(2.7)$ \\
Inferior + lateral wall & $12(2.23)$ \\
\hline
\end{tabular}

The clinical presentations were chest pain $(96.4) \%$, shortness of breathing (55\%), palpitation (15.8) \%, dizziness $5 \%$, syncope (2.4) \%, sweating (4.2)\%, nausea (3.1)\% and vomiting (2.6)\% in STEMI patients. While common clinical symptoms shortness of breath (100)\% present in NSTEMI followed by chest pain $(86) \%$, palpitation (11.9)\% dizziness $(15.2) \%$, syncope $(6.8) \%$, sweating $(11.8) \%$, nausea $(5) \%$, and vomiting $(3.3) \%$ which were more details in Table3.

Table 3. Clinical characteristics of STEMI and NSTEMI patients.

\begin{tabular}{llll}
\hline $\begin{array}{l}\text { Clinical } \\
\text { characteristics }\end{array}$ & $\begin{array}{l}\text { STEMI } \\
(\mathrm{N}=536) \%\end{array}$ & $\begin{array}{l}\text { NSTEMI } \\
(\mathrm{N}=59) \%\end{array}$ & $\begin{array}{l}\mathrm{P}- \\
\text { value }\end{array}$ \\
\hline $\begin{array}{l}\text { Shortness of } \\
\text { breath }\end{array}$ & $295(55)$ & $59(100)$ & $\mathrm{P}<0.05$ \\
$\begin{array}{l}\text { Chest pain } \\
\text { Palpitation }\end{array}$ & $517(96.4)$ & $51(86)$ & $\mathrm{P}<0.05$ \\
Dizziness & $27(15.8)$ & $7(11.9)$ & - \\
Syncope & $13(2.4)$ & $4(6.8)$ & - \\
Sweating & $23(4.2)$ & $7(11.8)$ & $\mathrm{P}<0.05$ \\
$\begin{array}{l}\text { Nausea } \\
\text { Vomiting }\end{array}$ & $17(3.1)$ & $3(5)$ & - \\
\hline
\end{tabular}

The comparison of risk factors between males and females and found the most common risk factors as hypertension (59) \% in males and $40.6 \%$ in female, while DM $(69.5) \%$ in males and $(30.4) \%$ in female at considering significant $p<0.05$. In addition, results showed smoking (70.8) \% in males and $(29.1) \%$ in females which was significant (Table-4). However, 
dyslipidemia present (48.6) \% in males and (47.4) \% in females but statistically were not significant.

\section{DISCUSSION}

There was no previous study regarding the Chinese population with clinical characteristics and risk factors associated with acute myocardial infarction in Hunan province China. In the present study found total 595 patients suffering acute myocardial infarction among 536(90\%) patients shown ST- segment elevation acute myocardial infarction (STEMI) and 59(9.9\%) non-ST segment elevation acute myocardial infarction (NSTEMI) which was revealed by a review of ECG. Besides, there was reported that in china most non-communicable disease consider as STEMI and subsequently needed to improve the protocol for the management. ${ }^{12}$ While this study also emphasis to arrange adequate hospital capacity and as well as train up the professionalism in their specialized field which leads to improving the patients caring.

Table 4. Clinical characteristics with risk factors among male and female patients.

\begin{tabular}{lllcl}
\hline Variables & Over all\% & Male $(\mathbf{n}=\mathbf{4 2 2}) \%$ & Female(n-=173)\% & P value \\
\hline Hypertension & $401(67.3)$ & $238(59)$ & $163(40.6)$ & $P<0.05$ \\
Smoking & $419(70.4)$ & $297(70.3)$ & $122(29.1)$ & $P<0.05$ \\
DM & $345(57.9)$ & $240(69.5)$ & $105(30.4)$ & $P<0.05$ \\
Dyslipidemia & $367(61.6)$ & $193(48.6)$ & $173(47.4)$ & -
\end{tabular}

In the present study, analysis of the clinical presentation of all the participants of STEMI and NSTEMI which were found chest pain $96.4 \%$ higher in STEMI than $86 \%$ in NSTEMI, while the shortness of breath $100 \%$ present in all NSTEMI patients than $55 \%$ in STEMI. On the other hand, sweating $4.2 \%$, nausea $5 \%$ and vomiting $3.3 \%$ present in NSTEMI patients versus to $4.2 \%, 3.1 \%$ and $2.6 \%$ shown in STEMI patients which were higher in NSTEMI. Although, the symptoms of palpitation $15.8 \%, 11.9 \%$ and syncope $2.4 \%, 6.8 \%$ found in STEMI and NSTEMI patients respectively. Smoking is the most common risk factor for AMI in this study, overall found $70.4 \%$ which compared between males and females and shown significantly higher $70.3 \%$ in males than $29.1 \%$ in females.

It has been evidenced that smoking is a serious health issue and responsible for the development of AMI in the turkey population and as well as globally. ${ }^{13}$ Another study, reported smoking is the most common risk factor among younger AMI patients. ${ }^{14,15}$ It has been reported that smoking may increase risk of AMI incidence as compared with non-smoking subjects. ${ }^{11}$ In addition, smoking perhaps thrombus formation due to impaired the homeostasis pathway to higher the oxidative stress that may mechanism of smoking cause cardiovascular diseases. ${ }^{16}$ Thus above this statement revealed that cessation of smoking may prevention of AMI. The current study found, the presence of hypertension increased in risk male AMI $59 \%$ compared to female $49.6 \%$. Although, previously documented that hypertension $35 \%$ found in male which was lower and $53 \%$ in female, which were higher than present results. ${ }^{16}$ However, present results indicated hypertension was an individual risk for STEMI patients, similarly reported by another study. ${ }^{17}$ Besides, diabetes mellitus found (69.5) \% in males compared to females $30.4 \%$ and highly significant. However, DM along was a common risk factor for males in our study, DM may leading cause of worse the AMI. ${ }^{18}$ Recently, DM is the most common health issue in the Asian population.

The DM and AMI has strong co-relation had been noticed in Europe and USA beside of Asian. ${ }^{19}$ In the present study shown dyslipidemia $48.6 \%$ in male and $47.4 \%$ in the female that was similar to another study. ${ }^{20}$ The high LDL and Low HDL has established as a risk factor for atherosclerosis and as well as cardiovascular diseases, while the present finding shown the mean LDL and HDL was $2.2 \pm 0.8 \mathrm{mmol} / \mathrm{L}$ and $1.3 \pm 0.3 \mathrm{mmol} / \mathrm{L}$ in all participants. Besides, in the present study, the anterior wall (51.3) \% and the inferior wall (41.9) \% was the most common side of infraction that similar to another study. 21 Nevertheless, it was well recognized that the infraction in anterior wall considers as a worse prognosis than inferior wall infarction. However, the present study evaluated the associated risk factors among the Chinese population in Hunan province, China. 


\section{CONCLUSION}

This retrospective study found STEMI was the most common type of AMI. In addition, chest pain and shortness of breath was the most common clinical feature in both STEMI and NSTEMI groups. In the present study noted most of the AMI patients were males than females with correlated risk factors of smoking, hypertension, diabetes mellitus and dyslipidemia. Therefore, the current study suggested about early diagnosis and prevention of the major risk factors may reduce the rate of AMI.

\section{Limitation}

In the present study have some limitation like a single study center, small sample size, and missing some data in some patients. However, need to further study with larger sample size in multihospital.

\section{CONFLICT OF INTEREST \\ None}

\section{ACKNOWLEDGEMENT}

Thanks to Professor Yang and Xie Ke of Xiangya Hospital, Cardiovascular unit for their help.

\section{REFERENCE}

1. Yusuf S, Reddy S, Ounpuu S etal. Global burden of cardiovascular diseases, part I: general considerations, the epidaemiologic transition, risk factors, and impact of urbanization. Circulation. 2001; 104:274653.

2. Cao CF, Ren JY, Zhou XH etal. Twenty-year trends in major cardiovascular risk factors in hospitalized patients with acute myocardial infarction in Beijing. Chin Med J. 2013; 126:4210-5.

3. Yang G, Wang $Y$, Zeng $Y$, et al. Rapid health transition in China, 1990-2010: findings from the Global Burden of Disease Study 2010. Lancet. 2013; 381:1987-2015.

4. Chen WW, Gao RL, Liu LS, et al. China cardiovascular diseases report 2015: a summary. J Geriatr Cardiol. 2017; 14:1-10.

5. GBD 2017 Causes of Death Collaborators. Global, regional, and national age-sexspecific mortality for 282 causes of death in 195 countries and territories, 1980-2017: a systematic analysis for the Global Burden of Disease Study 2017. Lancet. 2018; 392:1736-88.
6. Lim SS, Vos T, Flaxman AD, et al. A comparative risk assessment of burden of disease and injury attributable to 67 risk factors and risk factor clusters in 21 regions, 1990-2010: a systematic analysis for the Global Burden of Disease Study 2010. Lancet. 2012; 380:2224-60.

7. Zhang G, Yu C, Zhou M, et al. Burden of Ischemic heart disease and attributable risk factors in China from 1990 to 2015: findings from the global burden of disease 2015 study. BMC Cardiovasc Disord. 2018; 18:18.

8. Sehestedt T, Hansen TW, Li Y, et al. Are blood pressure and diabetes additive or synergistic risk factors? Outcome in 8494 subjects randomly recruited from 10 populations. Hypertens Res. 2011; 34:71421.

9. Serap Tutgun Onrat, 0 nder Akci , Zafer So ylemez etal Prevalence of myocardial infarction polymorphisms in Afyonkarahisar, Western Turkey. Mol Biol Rep. 2012; 39:9257-9264.

10. Gajalakshmi V, Peto R, Kanaka TS etal. Smoking and mortality from tuberculosis and other diseases in India: retrospective study of 43000 adult male deaths and 35000 controls. Lancet. 2003; 362:507-15.

11. Yusuf S, Hawken S, Ounpuu S, et al. Effect of potentially modifiable risk factors associated with myocardial infarction in 52 countries (the INTERHEART study): casecontrol study. Lancet. 2004; 364:937-52.

12. Jing Li, Xi Li, Qing Wang, Shuang Hu etal. ST-segment elevation myocardial infarction in China from 2001 to 2011 (the China PEACE-Retrospective Acute Myocardial Infarction Study): a retrospective analysis of hospital data. Lancet. 2015; 31: 385(9966): 441-451.

13. Nazif Aygut, Kurtulufl ozdemir, Adnan Abac et al. Prevalence of risk factors of ST segment elevation myocardial infarction in Turkish patients living in Central Anatolia. Anadolu Kardiyol Derg. 2009; 9: 3-8.

14. $\quad \mathrm{v}$ on Eyben FE, Bech J, Madsen JK etal. High prevalence of smoking in young patients with acute myocardial infarction. $J R$ Soc Health. 1996; 116(3):153-6.

15. R umboldt $Z$, Rumboldt $M$, Pesenti $S$ etal. Peculiarities of myocardial infarction at 
young age in Southern Croatia. Cardiologia. 1995; 40(6):407-11.

16. Ambrose JA, Barua RS. The pathophysiology of cigarette smoking and cardiovascular disease: an update. J Am Coll Cardiol. 2004; 43(10):1731-7.

17. Muhammad S Khan, Fahim H Jafary, Azhar $M$ Faruqui etal. High prevalence of lack of knowledge of symptoms of acute myocardial infarction inPakistan and its contribution to delayed presentationto the hospital. BMC Public Health. 2007, 7:284.

18. S tones $\mathrm{PH}$, Muller JE, Hartwell T et al. The effect of diabetes mellitus on prognosis and serial left ventricular function after acute myocardial infarction: contribution of both coronary disease an diastolic left ventricular dysfunction to the adverse prognosis. J Am Coll Cardiol. 1989; 14:49.

19. Woods KL, Samanta A, Burden AC. Diabetes mellitus as a risk factor for myocardial infarction in Asians and Europeans. Heart. 1989; 62(2):118-22.

20. Singh PS, Singh G, Singh SK. Clinical profile and risk factors in acute coronary syndrome. J Ind Acad Clin Med. 2013; 14(2):130-2.

21. Hafeez S, Javed A, Kayani AM. Clinical profile of patients presenting with acute ST elevation myocardial infarction. J Pak Med Assoc. 2010; 60(3):190-3. 